\title{
Context-Aware GRID Services: Issues and Approaches
}

\author{
Kerry Jean, Alex Galis, and Alvin Tan \\ University College London, Electrical Engineering Department, \\ Torrington Place, London, WC1E 7JE, U.K.; Tel: +44-20-7679573; \\ $\{$ kjean,agalis,atan\}@ee.ucl.ac.uk
}

\begin{abstract}
While Grid services introduce efficiency in addressing the changing and often large resource demands of Grid applications, making the system context-aware takes the approach a step further by incorporating the important aspects of customisation and personalisation into Open Grid Services Architecture (OGSA) services. This is achieved by extending the concept of virtual organisations (VO) to Grid context, which link context information to users, policies and resources. Context is defined as any information that can be used to characterise the situation of an entity. When this information is modelled and properly managed, the OGSA services can become context-aware and context-aware services can be offered by the Grid.
\end{abstract}

\section{Introduction}

Large amounts of computational power and storage are required for solving complex scientific and engineering problems [1]. The advent of the Internet has caused a proliferation in the growth of computers but much of this computing power is not fully utilised most of the time. The Grid concept seeks to harness this latent computing power by providing technology that enables the widespread sharing and coordinated use of networked computing resources to solve complex computing tasks $[1,2]$. A Grid is a geographically distributed platform, accessible to their users via a single interface, thus providing massive computation power and storage for the solving of complex tasks [3].

Grids provide coordinated sharing of computing resources like memory, software, data, processing power and other resources that are required for collaborative problem solving. This sharing arrangement is highly controlled with clearly defined rules as to what is shared, who is allowed to share and the conditions under which this sharing takes place. The set of individuals and/or institutions that abide by these rules is termed a virtual organisation (VO) [4]. Virtual Organisations supply a "context" ((the definition of context here is one of association) for operation of the Grid that can be used to associate users, their requests, and resources. These "contexts" can be used by services as an assertion of identity, e.g. this user or resource, is a member of this VO. This "context" can be extended to take into account any information that can be used to characterise Grid entities. This rich context information when modelled and managed introduces context-awareness into the Grid and enables both the customisation of the Open Grid Services Architecture (OGSA) services [6] and the provision of context-aware services (CAS) by the Grid to end users. 
The development of Grid computing was found on a basic architectural premise: a computing service platform is used as the means to interconnect intelligent endsystems to support large-scale applications. Most of the current research into Grid focuses on the Grid services architecture [6,7] or the development of Grid tools and protocols [1]. Other research has focussed on the management of the Grid [10,11,12] and the use of the Grid to solve complex scientific problems [9].

The ability of the Grid infrastructure to adapt to new functional requirements has become difficult to achieve. In addition, the current Grid computing infrastructure has insufficient flexibility, support for multiple applications and rapid deployment of applications on the infrastructure. With this in mind, it has been proposed to introduce context management into the Grid to make the OGSA services and by extension the Grid context-aware.

This paper presents the authors' position on context-awareness in Grids and will introduce the idea of context-awareness in the Grid; first enabling the OGSA services to become context-aware and eventually the provision of context-aware services (CAS) by the Grid. The next section will introduce the OGSA while the third section will define context and context management. Then the context Grid service will be detailed. The paper will conclude with a summary and the proposed work needed to create a context-aware Grid.

\section{Open Service Grid Architecture}

The establishment, management, and exploitation of dynamic, distributed heterogeneous VOs require an enabling service architecture for Grid systems and applications. Such an architecture is the Open Grid Services Architecture (OGSA) defined by the Open Grid Services Architecture Working Group (OGSA-WG) [6] of the Global Grid Forum [8]. The OGSA defines an integrated set of web service (WS)based service definitions designed both to simplify the creation of secure, robust Grid systems, and to enable the creation of interoperable, portable, and reusable components and systems via the standardisation of key interfaces and behaviours.

The OGSA defines, in terns of web services description language (WSDL) standard approaches to, and mechanisms for solving problems such as communicating with other services, establishing identity, negotiating authorisation, service discovery, and managing service collections. The three principal elements of the OGSA Platform are the Open Grid Services Infrastructure, the OGSA Platform Interfaces, and OGSA Platform Models [6].

Open Grid Services Infrastructure (OGSI) defines mechanisms for creating, managing, and exchanging information among entities called Grid services [7]. A Grid service is a web service that conforms to a set of conventions (interfaces and behaviours) that define how a client interacts with the Grid. These conventions, and other OGSI mechanisms, provide for the controlled, fault resilient, and secure management of the distributed and often long-lived state that is commonly required in distributed applications.

OGSA Platform Interfaces build on OGSI mechanisms to define interfaces and associated behaviours for various functions not supported directly within OGSI, such as service discovery, data access, data integration, messaging, and monitoring. 
OGSA Platform Models support these interface specifications by defining models for common resource and service types.

The OGSA Platform components are supported by a set of OGSA Platform Profiles addressing issues such as the following [6].

Protocol bindings: These work toward attaining interoperability among different Grid services by defining common mechanisms for transport and authentication. An example of such a Grid service transport profile is "SOAP over HTTP".

Hosting environment bindings: Profiles of this sort enable portability of Grid service implementations. For example, an "OGSA J2EE Profile" might define standardised Java APIs to allow the portability of Grid services among OGSI-enabled J2EE systems.

Sets of domain-specific services: These profiles define interfaces and models to address the needs of specific application domains. For example, an "OGSA Database Profile" might define a set of interfaces and models for distributed database management.

\section{Context and Context-A ware Services}

Context is defined as any information that can be used to characterise the situation of an entity, where an entity can be a person, place, physical or computational object. From this point of view, almost any information that occurs in the surroundings of a specific system usage can be termed context (e.g. the current time and date, the usage history, the user profile, the temperature at the user location, the position of the user, etc.). A context element is an autonomous component that also can be used by a number of application services, but that exists independently of the application service. Typical examples are: (1) calendar information (e.g. activity type, time, actors, location, etc.); (2) user location information (e.g. outdoors/indoors, building, room, etc.); (3) weather information (e.g. outside temperature, whether it is sunny, raining, snowing, etc.); (4) social context (e.g. role-student/staff/faculty; wife; friend; boss; colleague, director, etc.); (4) personal preferences (e.g. food preferences, favourite sports, etc.); (5) permission profiles (e.g. what services can contact the user and when, what contextual information can be made available to different types of services and under which circumstances); (6) user's behaviour (e.g. task, habits); (7) device and network characteristics (e.g. network elements, bandwidth).

Context-awareness cannot be achieved without an adequate methodology and a suitable infrastructure. There has been little work on the management of context within the Grid or on efforts to make the Grid context-aware. But there has been some work on CAS and its development $[13,14]$. However, this work has been hampered by the need to develop a custom context infrastructure for each application. Presently, there is an initiative to create an active, programmable platform for the creation and delivery of context aware services in the Context project [15]. The introduction of context-awareness into the Grid will easily enable the personalisation and customisation of OGSA services. These services will easily adapt to a change in their context. When OGSA services become context-aware, the Grid could then offer context-aware services to customers. Hence, context-awareness will enable new types of services in Grid computing environments. 
One of the most difficult aspects to cater in putting context in the Grid is that the context of people, networks, and applications can be time varying and sometimes can vary quite rapidly. This poses the challenge of tracking different types of environment changes in real time and also to react accordingly to these changes [14]. This is a key feature of context-aware applications and systems so as to fully exploit the richness of context-information. In view of this, we have proposed a flexible Grid context service that fits neatly into the Grid service architecture that was discussed in the previous section. Our proposal, which will be discussed in the next section, entails defining a new OGSA service, a Grid context service to manage context and make the Grid context aware.

\section{Grid Context Service}

In order to make a Grid context-aware, we introduce the context service into the OGSA. This is an extension of the virtual organisation's concept of associating users and resources to VOs by supplying a "context" (the definition of context here is one of association). This concept is extended by looking at "context" as not just an association of users or resources but as any information that characterises the situation of an entity be it associations, relationships or characteristics. Hence, the context Grid service is as an extension of this VO "context" linking contextual information, users, resources and policies. This enables the creation of services that are context-aware, and hence, easily customisable and personalisable.

The context Grid service creates a general framework for the gathering, storage and management of context information as well as an infrastructure for the creation and deployment of CAS offerings to end users. The context service is implemented through a host of services. The context management service ensures the efficient gathering, storage, transport and timely use of context information inside the Grid. Through interaction with the context information management service, the OGSA services become context-aware and hence their context can be used to make them easily customisable and personalisable. CAS management is concerned with the definition, deployment and operation of CAS by the Grid. It uses the context management service and other OGSA services to enable the Grid to offer CAS to end users, thus increasing the scope of the services that the Grid can offer.

\subsection{Context Management Service}

The context management service uses the context gathering and publishing service, the context retrieval service and the context storage service. Context information is obtained from a wide plethora of applications, services and sensors (collectively called context sources) spread all over the network. The job of the context gathering service is to collect this information and put it into a common model understood and accessible by all components of the context service. This highly distributed characteristic introduces a great challenge for the manageability of all this information. Hence there is a need for a common context information model and a context publishing protocol. The context is then stored in a context information base for access by context consumers through the context retrieval service. The relationship between the context sources and consumers are shown in Fig. 1. below. 


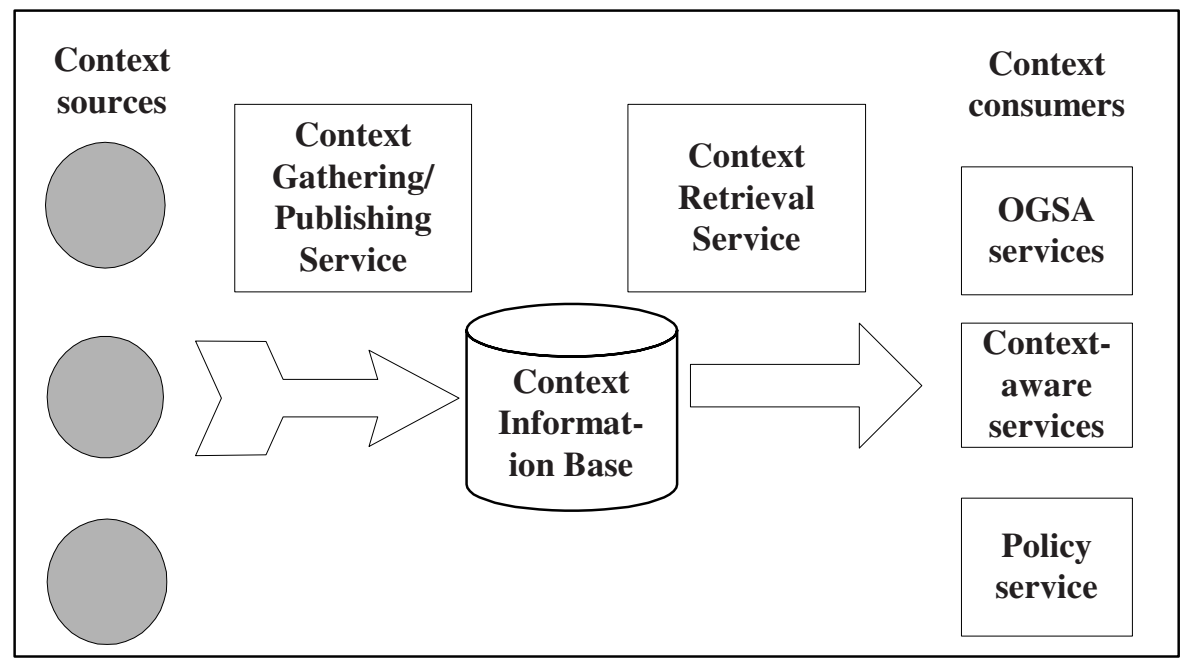

Fig. 1. Relationship between context sources and context consumers

The interaction between context consumers and context sources could be described as follows: (1) in a push mechanism, context sources periodically push updated context information to the context management service. This information is maintained in a context information base and client inquiries are serviced from a local store. (2) In a pull mechanism, the context service must explicitly request context information. It can either make this request on a periodic basis (polling) or when an application demand arises. A push system collects data ahead of need and thus may offer better performance. However, it may consume substantial resources transferring and storing information that is never required. A pull system may use fewer resources by obtaining only the data that is required. However, this exposes the context service to inevitable network delays and unavailability.

\subsection{Context Information Modelling}

A context information model must be defined in order to map context information, for common gathering, storage and parsing. The context information model must be rich and flexible enough to accommodate not only the current but also future aspects of context information. The model should scale well and be based on standards as much as possible. The context model proposed is based on an entity and relationship approach. This model is easily translated into an object-oriented information model and fits well into object-oriented programming (OOP). An entity is composed of a set of intrinsic characteristics or attributes that define the entity itself, plus a set of relationships with other entities. Context is classified into four main types of entities: personal context, place context, object context and task context. These main entities form the base entity classes. Subclasses of these entities are defined and are called sub-entities. The syntax of both entities and sub-entities are defined in XML. 


\subsection{Context-Aware Management Service}

Grid CAS management facilitates the management and provision of CAS by linking providers of Grid resources to appropriate context information via predefined policies or agreements. Users are associated with the CAS as a means to exploit those Grid resources. The CAS management service provides a set of basic service components for the creation, lifetime management and termination of CAS. They include, as shown in Fig. 2 below; the CAS creation/destruction service, the CAS registry service, the subscription service and a policy-based CAS lifetime management service. For successful operation, these services rely on the context management service for their context information and other OGSA services especially the policy and agreements service.

The CAS creation/destruction service deals with the definition of the CAS, their deployment and their termination. CAS are created by the composition or customisation of predefined basic services stored in policy format. This composition mechanism is driven by a creation policy. The subscription/customisation service deals with customer subscription and service customisation. The CAS registry service keeps a directory of all the CAS that have been defined by the Grid context service. The policy-based service lifetime management manages the services as they operate. It provides the extensibility, easy management and operability that CAS require to be functional and efficient.

The Grid context service makes great use of the OGSA policy and agreement services. As stated earlier, policies are used to specify the requirements for creation and management of CAS. These policies may contain information that describes how to create and manage the CAS as well as how these services respond to changes in context and how they behave under specific conditions. A policy-based manager is used for service management while basic CAS are stored in policy format. Policy decision and enforcement points are also implemented. Hence, the context Grid service can be implemented using policy-based management to manage the gathering, publishing, storage and retrieval of context information as well as for the creation and management of CAS.

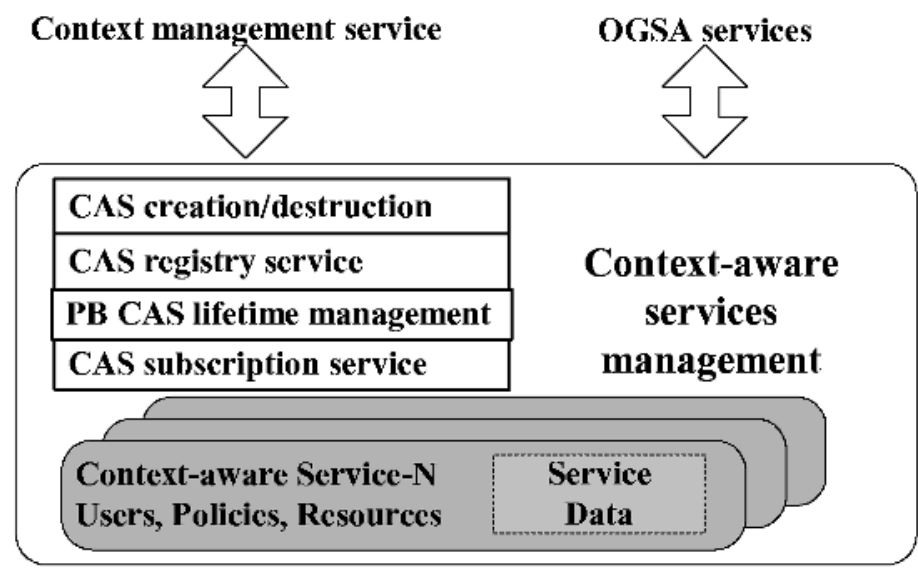

Fig. 2. GRID Context-aware Management Services 


\section{Conclusions and Future Work}

Grid technology enables widespread sharing and coordinated use of networked computing resources. Grids are large, distributed, dynamic and heterogeneous platforms used for collaborative problem solving. By adding context-awareness to the Grid infrastructure, we expect to add the extra dimension of easily personalisable and customisable context-aware OGSA services. Such a Grid can then offer context-aware end services, which are very relevant today especially with the proliferation of mobile devices and in context rich situations. This is achieved through the introduction of the context Grid service.

As future work, protocols must be defined for interactions between the Grid, the context information management service, the context-aware services management services and the policy service. Further work must also be done on policy-based management of the context service. There is ongoing work on the creation of an active policy based system for the efficient creation of context-aware services in the Context project [15]. The results of this initiative are eagerly awaited and will be used as a guideline to implement context-awareness in the Grid. A proof of concept of the context Grid service will be provided with the future implementation of a contextaware virtual private Grid. However, there is also a need to investigate alternatives for context information management architectures and context Grid service implementations.

Acknowledgements. This paper partly describes work in progress in the EU IST project CONTEXT [15]. The IST programme is partially funded by the Commission of the European Union.

\section{References}

1. I. Foster, C. Kesselman, "Globus: A MetaComputing Infrastructure Toolkit", International Journal of Supercomputer Applications, pp. 115-128, Vol. 11 No. 2, 1997.

2. F. Berman, G.C. Fox, A. J. G. Hay, "Grid Computing", Wiley 2003.

3. I. Foster, C. Kesselman editors, "The Grid: Blueprint for a Future Computing Infrastructure", Morgan Kaufman, San Francisco, USA, 1999.

4. I. Foster, C. Kesselman, and S. Tuecke, "The Anatomy of the Grid, Enabling Scalable Virtual Organisations", International Journal of Supercomputer Applications, 2001.

5. D. B. Skillicorn, "Motivating Computational Grids", Proceedings of the Second IEEE/ACM International Symposium on Cluster Computing and the Grid, IEEE 2002.

6. I. Foster, D. Gannon, H. Kishimoto, editors, "The Open Grid Services Architecture Version 0.13a",

https: //forge.gridforum.org/projects/ogsa-wg/docman, Nov. 2003.

7. S. Tuecke et al, "Open Grid Services Infrastructure (OGSI) Version 1.0", Global Grid Forum Draft Recommendation, June 2003

http://www.globus.org/research/papers.html.

8. Global Grid Forum Web Page: http://www.gridforum.org.

9. EU DataGrid Project Web Page: http://www.eu-datagrid.org. 
10. J. Frey et al, "Condor-G: A Computational Management Agent for Multi-Institutional Grids", Proceedings of the Tenth IEEE Symposium on High Performance Distributed Computing (HPDC10), San Francisco, USA, 2001.

11. K. Yang, A. Galis, and C. Todd, "A Policy-Based Active Grid Management Architecture", Proceedings of the Tenth IEEE International Conference on Networks (ICON02), pp. 243248, IEEE, Singapore, 2002.

12. A. Galis, J. P. Gelas, L. Lefèvre, and Y. Yang, "Programmable Network Approach to Grid Management and Services", International Conference on Computational Science 2003, LNCS 2658, pages 1103-1113, Melbourne, Australia, June 2003, www. science.uva.nl/events/ICCS2003/.

13. Gregory D. Abowd et al, "Context-Awareness in Wearable and Ubiquitous Computing", First International Symposium on Wearable Computers, pp. 179-180, 1997.

14. P. Gray and D. Salber, "Modelling and Using Sensed Context Information in the Design of Interactive Applications", Proceedings of the Eighth IFIP Working Conference on Engineering for Human-Computer Interaction (EHCI'01), Toronto, Canada, May 2001.

15. CONTEXT Project website, http: // context. upc.es. 